\title{
Resting-state network mapping in neurosurgical practice: a review
}

\author{
Carl D. Hacker, MD, PhD, ${ }^{1}$ Jarod L. Roland, MD, ${ }^{2}$ Albert H. Kim, MD, PhD, ${ }^{1}$ \\ Joshua S. Shimony, MD, PhD, ${ }^{3}$ and Eric C. Leuthardt, MD'
}

\begin{abstract}
Departments of ${ }^{1}$ Neurological Surgery and ${ }^{3}$ Radiology, Washington University School of Medicine, St. Louis, Missouri; and ${ }^{2}$ Department of Neurosurgery, University of California, San Francisco, California
\end{abstract}

\begin{abstract}
Resting-state functional MRI (rs-fMRI) is a well-established method for studying intrinsic connectivity and mapping the topography of functional networks in the human brain. In the clinical setting, rs-fMRI has been used to define functional topography, typically language and motor systems, in the context of preoperative planning for neurosurgery. Intraoperative mapping of critical speech and motor areas with electrocortical stimulation (ECS) remains standard practice, but preoperative noninvasive mapping has the potential to reduce operative time and provide functional localization when awake mapping is not feasible. Task-based fMRI has historically been used for this purpose, but it can be limited by the young age of the patient, cognitive impairment, poor cooperation, and need for sedation. Resting-state fMRI allows reliable analysis of all functional networks with a single study and is inherently independent of factors affecting task performance. In this review, the authors provide a summary of the theory and methods for resting-state network mapping. They provide case examples illustrating clinical implementation and discuss limitations of rs-fMRI and review available data regarding performance in comparison to ECS. Finally, they discuss novel opportunities for future clinical applications and prospects for rs-fMRI beyond mapping of regions to avoid during surgery but, instead, as a tool to guide novel network-based therapies. https://thejns.org/doi/abs/10.3171/2019.9.FOCUS19656
\end{abstract}

KEYWORDS resting state; functional connectivity; functional mapping; fMRI

$\mathrm{P}$ RESERVATION of function and maximal extent of resection are inherently opposed objectives in the surgical treatment of brain tumors. Optimization of these competing objectives has significant implications for the clinical outcomes of our patients. The relationship between maximizing the extent of resection and a patient's overall survival has been extensively documented for glioma patients. ${ }^{23,24,26}$ However, a greater extent of resection risks a higher frequency and severity of functional deficits, which, in the case of motor and language functions, have been associated with decreased overall survival ${ }^{17,31,44}$ and worsened quality of life. ${ }^{22}$ This balance is especially precarious in cases of glioblastoma resection in which extensive (> 98\%) resection must be achieved to affect survival. ${ }^{48}$

Functional mapping techniques in neurosurgery were first developed in the context of epilepsy surgery but were soon adopted in the surgical treatment of gliomas and vascular malformations. . $, 20,35$ Intraoperative electrocortical stimulation (ECS) mapping remains the standard of care for precise and reliable identification and preservation of brain areas critical to speech and motor functions. ${ }^{36}$ However, intraoperative mapping comes at a significant burden in operating room time and modifications to anesthesia, particularly when operating on the brain of an awake patient. Preoperative functional mapping strategies using advanced neuroimaging techniques can assist in the identification of cases most appropriate for awake mapping and facilitate direct stimulation to areas of greatest interest.

Functional MRI (fMRI) is a widely used technique for preoperative mapping. fMRI measures the oxy/deoxyhemoglobin ratio as a contrast mechanism, sampled sequentially over time, to produce the blood oxygen level-dependent (BOLD) signal. In a task-based paradigm, as has been typically used in clinical practice, motor or language tasks are employed in a block design, and task responses are averaged to produce maps of significant activation..$^{18,25}$ In some centers, task-based fMRI has supplanted invasive procedures such as Wada testing for language localization. ${ }^{51}$ However, task-based fMRI is limited by many factors affecting patient participation, such as age, cognitive impairment, neurological deficits, and poor cooperation.

ABBREVIATIONS BOLD = blood oxygen level-dependent; ECS = electrocortical stimulation; fMRI = functional MRI; MLP = multilayer perceptron; rs-fMRI = resting-state $\mathrm{fMRI} ; \mathrm{RSN}$ = resting-state network; TMS = transcranial magnetic stimulation.

SUBMITTED August 1, 2019. ACCEPTED September 12, 2019.

INCLUDE WHEN CITING DOI: 10.3171/2019.9.FOCUS19656. 
Task-based fMRI is also inherently limited in that it can only map components of brain functions that are represented by task paradigms and generally is only used to map a single function at a time.

Many of the limitations of task-based fMRI are mitigated by the use of resting-state fMRI (rs-fMRI). Resting-state fMRI is based on the analysis of spontaneous very low-frequency $(<0.1 \mathrm{~Hz})$ BOLD signal fluctuations, specifically the spatial patterns of correlations in signals across the brain. ${ }^{13}$ These analyses are performed with the understanding that widely distributed brain regions that are involved in similar functions demonstrate synchronous low-frequency fluctuations when the brain is not performing any type of cognitive, motor, or language task. This phenomenon was first noted in 1995 by Biswal et al., where subjects performed a hand-movement task, activating sensorimotor regions when analyzed by traditional fMRI methods. ${ }^{4}$ Resting-state fMR images were subsequently analyzed (while the subjects were at rest), and it was noted that regions of task activation in the sensorimotor cortex were found to have a high degree of temporal correlation. Further studies demonstrated that spatially correlated BOLD signal activity recapitulates task-based fMRI responses to a wide variety of behavioral paradigms. ${ }^{47}$ Notably, all networks can be analyzed from the same data set, making this method highly efficient compared to task paradigms. Furthermore, imaging for resting state can be performed under sedation, and the results are inherently independent of task performance.

Resting-state fMRI is well established in the scientific literature and is emerging as a useful tool for clinical brain mapping. ${ }^{45}$ In this review, we provide a summary of how resting-state networks (RSNs) are defined, their emerging clinical utility and limitations, and prospects for future clinical applications.

\section{Defining Networks by rs-fMRI}

RSNs have predominately been computed from fMRI data by one of two methods: seed-based correlation mapping $^{5}$ or spatial independent component analysis. ${ }^{1}$ Independent component analysis separates signals by maximizing statistical independence; in practice, this means finding clusters of signals within the brain (i.e., networks) that are maximally distinct from each other. RSNs obtained by spatial independent component analysis are theoretically unbiased by prior assumptions. Seed-based correlation mapping operates by first choosing a region in the brain and then finding other brain regions with signals most correlating to the point of interest. Repeating this process for a seed versus every point in the brain produces a correlation map, and in turn, this can be repeated for numerous seeds throughout the brain to examine their connectivity. This method of analysis is clearly biased by the choice of seed regions. However, many strategies have been devised to achieve independence from prior assumptions by systematically analyzing seeds placed throughout the brain. ${ }^{52}$ A dense sampling with numerous seeds can then be analyzed using graph theory ${ }^{39}$ or clustering methods, ${ }^{28,54}$ yielding RSN topographies that are effectively independent from priors.
Methods for defining networks described above, such as independent component analysis and clustering, are excellent for discovering new features in data and have been instrumental in defining RSNs throughout the brain over the last 15 years. In the context of machine learning, these methods collectively are considered "unsupervised" methods for analyzing data. However, in clinical application, specifically in preoperative planning for neurosurgery, we aim to define very specific networks and brain regions that are critical to preserving function and to do so in a manner that is reliable and clinically feasible. In general, unsupervised methods are ill-suited for this task, and the reasons for this relate to the structure of networks in the human brain.

A fundamental assumption in the analysis of brain networks is that more strongly correlated brain areas are more closely related in function and therefore have greater "functional connectivity." Multiple highly connected brain regions make up functional modules that are relatively sparsely connected to regions in other modules. This happens on a range of spatial scales in a scale-free manner, ${ }^{50}$ organized as a modular hierarchy where, within each module, there will be a set of submodules and, within each submodule, a set of sub-submodules, and so on. ${ }^{32,39}$ Just as one can choose different sizes of branches to cut up a tree and still end up with all the leaves, one can "parcellate" the brain into different functional systems or areas at varying levels of granularity, while still producing a faithful representation of the functional organization of the brain (compare Yeo et al., ${ }^{54}$ Lee et al., ${ }^{28}$ and Power et al..$^{39}$ for examples). To illustrate, a greater rs-fMRI correlation between Broca's area and Wernicke's area implies that these areas are more functionally similar than Broca's area and the primary visual cortex, and thus a putative language network might include Brodmann areas 44-45 (Broca) and Brodmann area 22 (Wernicke), but not Brodmann area 17 (V1). Of particular concern to neurosurgeons, an unsupervised method may unpredictably recover just Wernicke's area as a component, or it may return the entire language network in addition to other noneloquent associative areas of related functions, such as memory (specifically, default mode network components [see Power et al. ${ }^{39}$ and Lee et al. ${ }^{28}$ ]). Such inconsistencies stem from the fact that unsupervised learning procedures are ill-suited for constraint to a particular hierarchical scale; to be clinically feasible, some post hoc classification strategy (e.g., template matching) must be used to find a recovered component resembling the desired functional network.

In neurosurgery, our goal is fundamentally different from the large body of RSN literature in that our objective is not to discover RSNs or to study their functional relevance but rather to map the topography of known RSNs reliably in individual patients. This is well posed as a supervised learning problem of classification or regression. In prior work, a supervised classifier (multilayer perceptron [MLP]) was used to associate specific RSN identities with correlation maps generated from predefined seeds. ${ }^{19}$ Perceptrons are feed-forward artificial neural networks, originally modeled on the human visual system, trained to associate weighted sums of input features with predefined 

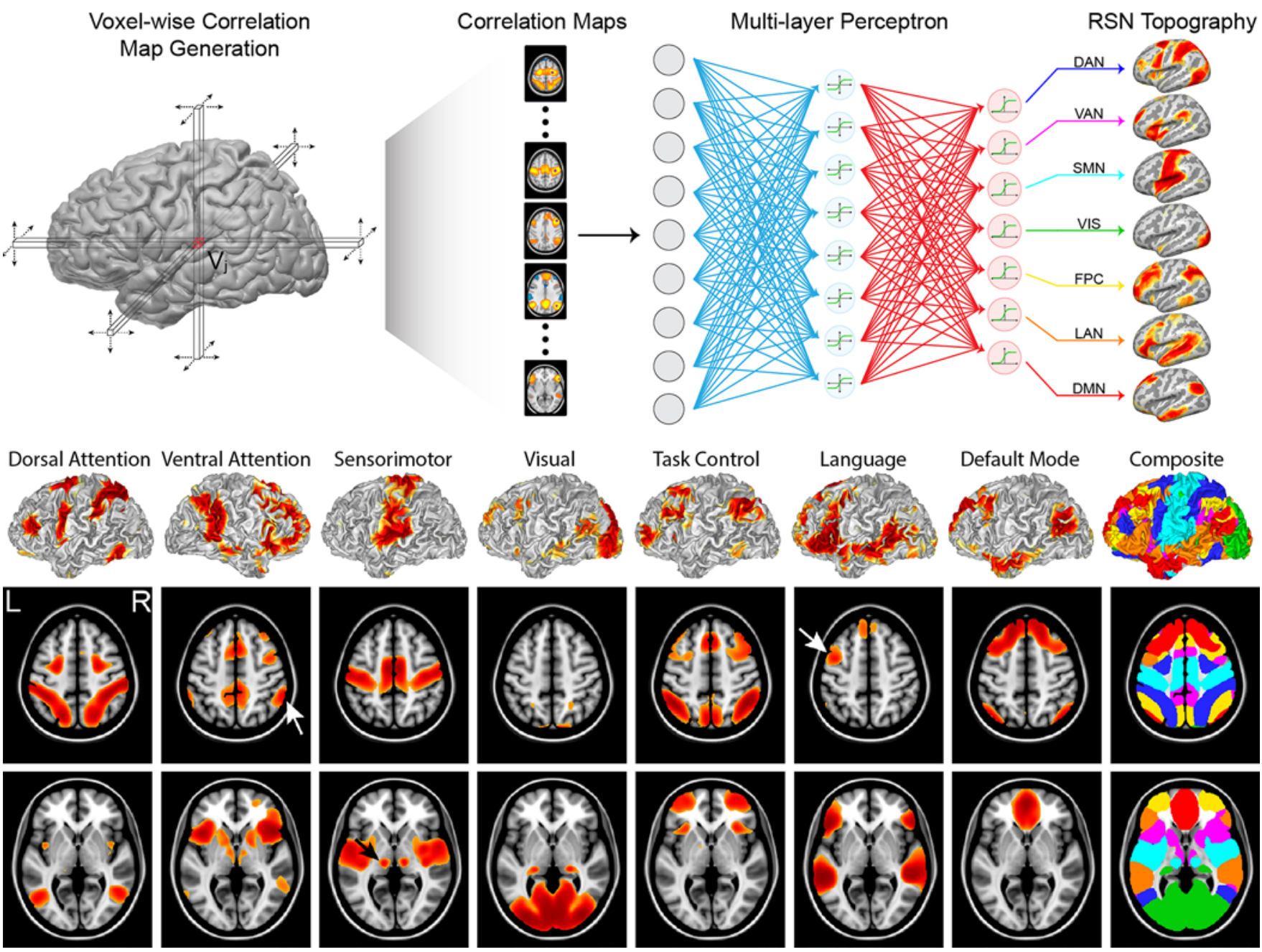

FIG. 1. Classification of resting-state data into RSNs using a neural network. Upper: Each brain locus is used as a seed to produce one correlation map that is passed through the neural network, giving an estimate of its membership in each RSN. This process is repeated for every locus in the brain, producing full-brain maps of network membership. Center: RSN topography for an individual subject projected onto a pial surface rendering. Lower: Volume-averaged RSNs across 692 participants show the typical topography of each network. Note the lateralized findings on the left for language and right for ventral attention networks (white arrows). DAN = dorsal attention network; DMN = default mode network; FPC = frontoparietal control network; LAN = language network; SMN = sensorimotor network; VAN = ventral attention network; VIS = visual network. Based on figures from Hacker CD, Laumann TO, Szrama NP, Baldassarre A, Snyder AZ, Leuthardt EC, et al: Resting state network estimation in individual subjects. Neuroimage 82:616-633, 2013; doi:10.1016/j.neuroimage.2013.05.108. Reproduced with permission of Elsevier.

output classes. ${ }^{43}$ Given enough training data and the proper design, MLPs are universal approximators, capable of learning any function or mapping. ${ }^{21}$ We trained an MLP to associate RSN identities with seed-based correlation maps generated in a large number of subjects using canonical network nodes. After training, correlation maps generated for every point in the brain can be classified to generate whole-brain maps of network identities (Fig. 1). Critically, classifier behavior (i.e., decision boundaries for which connectivity patterns represent given networks) is fixed after training; thus, results are guaranteed to represent the same entity (at the same hierarchical scale) across individuals or populations. Not only does this mitigate many of the challenges of bringing extant unsupervised resting-state methods into clinical use, but also a consistent method allows surgeons the opportunity to develop an intuition about the topographic variability of networks identified across patients and pathologies. Consistent RSN definitions also allow comparison to known anatomical landmarks. RSN mapping is frequently combined with tractography such that the sensorimotor RSN can be compared to the corticospinal tract and the language RSN to the arcuate fasciculus; see Roland et al. ${ }^{41}$ and case examples below. Agreement between these multiple modalities allows for increased confidence in the localization of critical functional regions.

\section{Performance of rs-fMRI}

It is difficult to assess the performance of resting-state mapping in the clinical setting, given the great heterogeneity in functional anatomy and pathology across patients, 
and, critically, due to the absence of definable "ground truth" in the localization of function. Ultimately, large retrospective reviews or potentially clinical trials comparing detailed neuropsychological testing to preoperative mapping results (in relation to lesion location and the extent of resection) will be required to directly establish the utility of functional imaging. Substantial work has shown correspondence between rs-fMRI networks and the topography of task-evoked responses. ${ }^{47}$ This has been specifically demonstrated in motor, language, and visual tasks; ${ }^{8}$ more recent studies have demonstrated that a common set of core resting state components underlie the patterns of brain activation in a large battery of 83 cognitive tasks. ${ }^{53}$ Of specific interest to neurosurgical application, Dierker et al. ${ }^{9}$ compared sensorimotor topography across resting-state, task-based, and anatomical definitions in 38 glioma patients. They found that both methods provided accurate representation of the sensorimotor cortex. However, the resting-state representation generally resulted in a better overlap with anatomically defined peri-Rolandic cortex because it yielded a more complete topography of an entire functional system, whereas task-based fMRI responses were more focal, that is, limited to a particular response, such as finger tapping.

Clinically, awake mapping with ECS is considered the gold standard. Systematic comparisons between ECS and rs-fMRI have demonstrated concordance in both adult ${ }^{33}$ and pediatric populations. ${ }^{42}$ Mitchell et al ${ }^{33}$ directly compared ECS and RSN maps at a range of thresholds to investigate the sensitivity and specificity of rs-fMRI for detecting critical regions as defined by stimulation. They found that rs-fMRI generally detected broader cortical regions than ECS, yielding good sensitivity but poor specificity. For example, the sensorimotor RSN encompasses both primary motor and primary sensory cortex, in addition to supplementary motor regions. ${ }^{4,19}$ ECS-positive sites only included primary motor cortex sites producing a response (precentral gyrus), whereas the resting-state motor network included the precentral and postcentral gyri and some insular cortices (note the false-positive regions in Fig. 2). Comparison between stimulation mapping for speech and the resting-state language network is more complex. Intraoperatively, ECS has been used specifically to define regions that produce speech arrest. However, language representation in the brain as identified by fMRI extends far beyond regions of speech generation per se. ${ }^{3,6}$ Furthermore, not all brain regions involved in speech generation may necessarily produce arrest when stimulated intraoperatively. Therefore, a highly sensitive technique is appropriate when followed by a more specific but costly (in terms of operating room time and potential patient morbidity) technique, such as awake stimulation mapping. The more clinically significant error is when a critical language site is misidentified as noncritical to function, which could contribute to an overly aggressive resection and an avoidable postoperative deficit. Mitchell et al. found that the likelihood that an electrode was misidentified as nonfunctional drops substantially between 1 and $2 \mathrm{~cm}$ from the border of the functional zone (Fig. 3). This accords with the margin of safety discussed in relation specifically to stimulation mapping. ${ }^{33}$

Roland et al..$^{42}$ demonstrated rs-fMRI for mapping the somatomotor network in a wide age range of pediatric patients who underwent cortical stimulation mapping of the sensory and motor cortex and found good agreement between the MLP and stimulation mapping. Preoperative mapping techniques are specifically advantageous in pediatric populations as these patients are often too young to participate in an awake craniotomy. The inability to follow commands adequately in the operating room may not only result in inadequate mapping but also risk anesthesia complications.

\section{Illustrative Cases Case 1}

This 33-year-old male patient with no past medical history presented with a several-month history of headache, nausea, and vomiting. He reported 4 months of headache, typically grade $3-4$ of 10 in severity. He also complained of intermittent nausea and vomiting of the same duration, which occurred approximately once per week. He described presyncope during these weekly episodes. $\mathrm{He}$ said that for the last 1.5 months, he had had occasional "visual auras," seeing lights and haziness around people. He denied ever having any seizures. He endorsed several years of left eye twitching but denied any focal weakness, numbness, or tingling. He was neurologically intact on examination. Brain MRI demonstrated a left, frontotemporal, heterogeneous, faintly enhancing lesion measuring up to $7.4 \times 6.7 \mathrm{~cm}$ in the axial plane and with $1.8 \mathrm{~cm}$ of midline shift. Advanced imaging demonstrated language network foci in the left frontal lobe near the lesion. The left arcuate fasciculus coursed inferior to the tumor and colocalized to language RSN regions. Awake surgery was not performed due to extensive mass effect. A left frontotemporal craniotomy was performed with the intention of leaving a small rind of tumor against Broca's area and the arcuate fasciculus; an $80 \%-85 \%$ resection was achieved. Postoperatively, the patient had no weakness or language deficits and was discharged on postoperative day 3. Pathological examination of the tumor specimen demonstrated a WHO grade IV, IDH-mutant glioblastoma.

\section{Case 2}

This 24-year-old male patient, who was otherwise healthy, presented to the emergency department after a motor cycle collision. He had sustained a blunt traumatic abdominal injury that required a splenectomy. A head CT scan demonstrated a hypodense lesion, prompting referral to neurosurgery. He was neurologically intact, and the lesion caused no symptoms. MRI demonstrated a T1-weighted hypointense, and FLAIR and T2-weighted hyperintense, nonenhancing lesion in the left frontal lobe (Fig. 4). The lesion was in close proximity to the frontal components (Broca's area) of the language network. The patient underwent an awake left frontal craniotomy and intraoperative stimulation for language mapping. He had no postoperative language deficit. Histopathological examination showed a WHO grade II oligodendroglioma with no $1 \mathrm{p} / 19 \mathrm{q}$ codeletion, loss of ATRX, elevated $\mathrm{p} 53$, and positive IDH1 R132H mutation. Interestingly, at the 3-year follow-up visit, the patient's language network had 
A

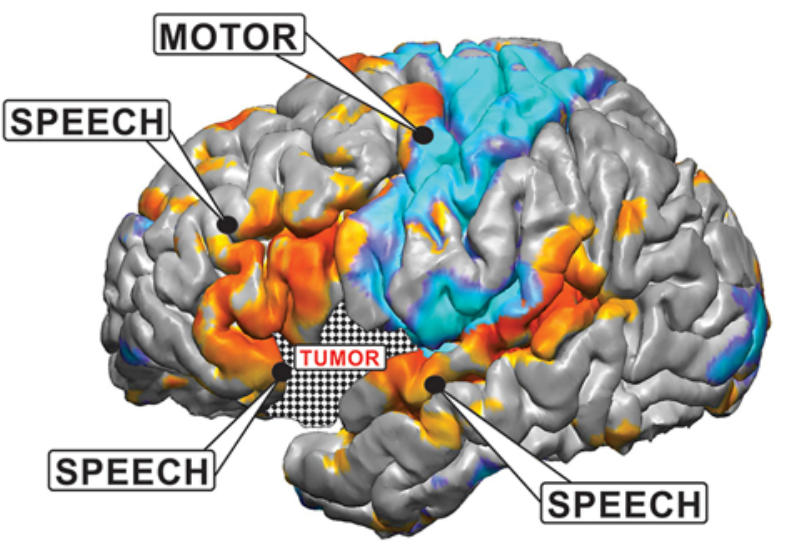

B

PROBABILITY OF A MISSED MOTOR ELECTRODE

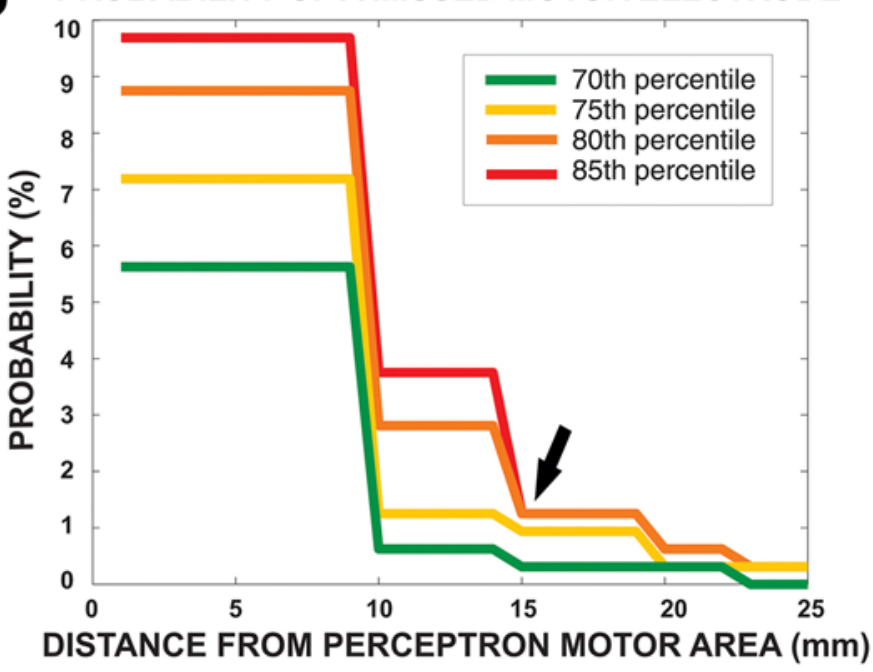

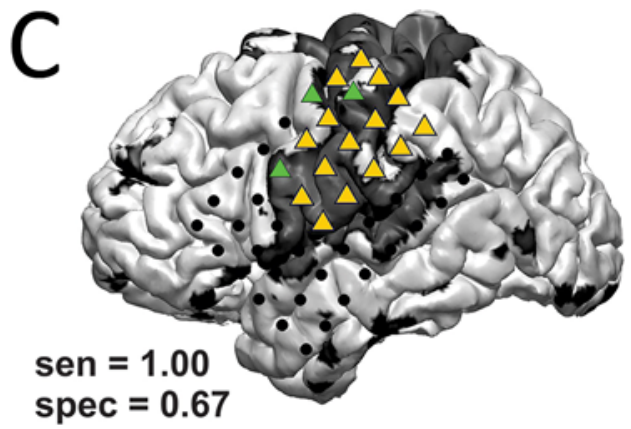

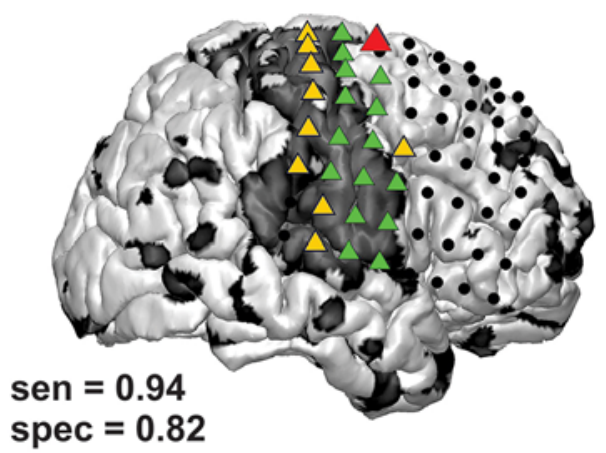

False Negative False Positive True Positive True Negative

FIG. 2. Relation of stimulation mapping to RSN mapping in adults. A: Stimulation-positive sites are labeled in relation to RSN topographies (cyan, motor; orange, language). B: Across a range of thresholds, the probability of rs-fMRI failing to identify an ECS-defined functional motor site falls dramatically 1-2 cm from the motor RSN. C: Concordance between motor RSN and stimulation sites. Note the increased specificity of ECS for the primary motor component (green triangles in the precentral gyrus) of the sensorimotor network (black shaded surface overall). Across a range of thresholds, the probability of rs-fMRI failing to identify an ECS-defined functional motor site falls dramatically $1-2 \mathrm{~cm}$ from the motor RSN. Panels B and C reproduced from Mitchell TJ, Hacker CD, Breshears JD, Szrama NP, Sharma M, Bundy DT, et al: A novel data-driven approach to preoperative mapping of functional cortex using resting-state functional magnetic resonance imaging. Neurosurgery 73:969-982, 2013. By permission of the Congress of Neurological Surgeons.

reorganized to a right-dominant pattern. This is significant in that RSNs are generally stable within and across imaging session ${ }^{27}$ but can show changes over timescales of months to years, ${ }^{38}$ and language network reorganization in patients with gliomas has been demonstrated previously with magnetoencephalography. ${ }^{49}$

\section{Limitations and Methodological Considerations}

The principal limitation to the use of rs-fMRI for preoperative mapping is the high degree of advanced imaging expertise currently necessary to process and interpret the images. Seed-based connectivity allows great flexibility in examining functional connectivity, but would require individualized seed placement in the presence of distorted anatomy, and produces a series of images that in aggregate are too complex to drive a surgical decision. RSNs derived from independent component analysis can be more easily automated, but as discussed above, a post hoc method must be used to identify components, and, more importantly, the recovered RSNs may not represent the same entity across patients. Approaches using supervised learning overcome these challenges, as described previously, but are inherently limited by the data set used in training. The method developed in Hacker et al. ${ }^{19}$ defined a language network based on a meta-analysis of seeds derived from language tasks. This is in contrast to speech-specific nodes mapped by intraoperative stimulation. A classifier could be specifically trained to localize regions associated with 

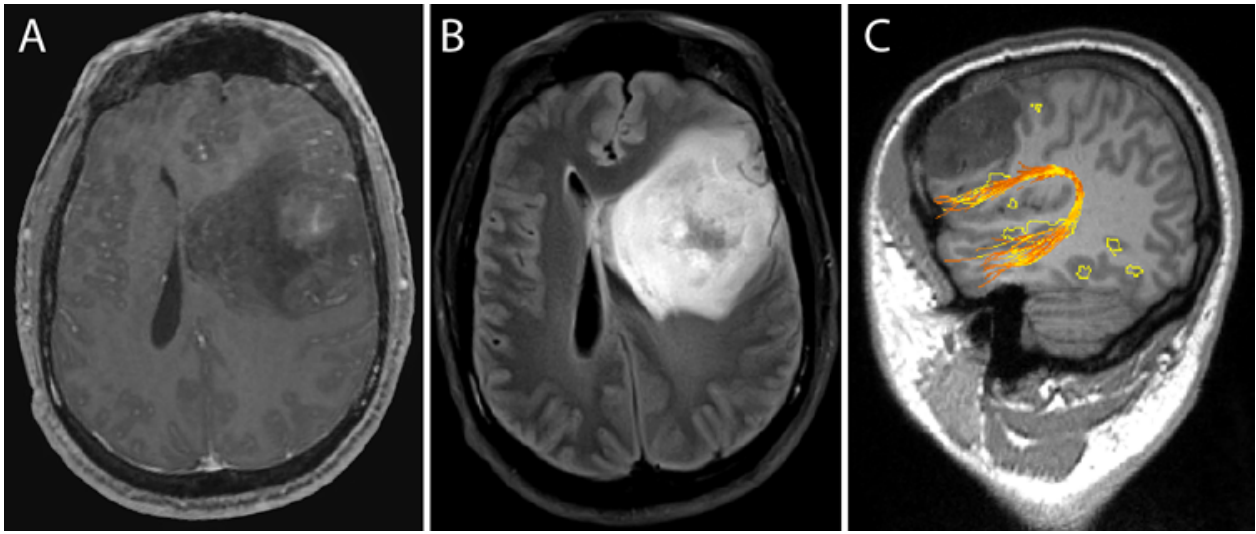

FIG. 3. Left frontotemporal glioblastoma. A: T1-weighted contrast-enhanced image demonstrating faint, heterogeneous enhancement. B: The lesion is T2 signal hyperintense with associated vasogenic edema. C: Advanced functional image demonstrating frontal language RSN regions posterior and inferior to the tumor. Arcuate fibers colocalize with both frontal and temporal language regions. The image in $\mathrm{C}$ was generated using a clinical workstation that is integrated with the intraoperative neuronavigation system.

speech arrest. Alternatively, the broader language network may define regions where more sensitive neurocognitive testing would be required to reveal a postoperative deficit. Large, prospective clinical trials with detailed pre- and postoperative neurocognitive assessments will be required to better establish the relationship between surgical lesions and cognitive deficits.

An important, practical consideration in clinical incorporation of rs-fMRI is the duration of scan time necessary for mapping RSNs. Gordon et al. ${ }^{15}$ specifically evaluated the effect of run length on the reliability of many functional connectivity measures in highly sampled subjects (including 5 hours of resting-state and 6 hours of taskbased fMRI) and found consistently poor reliability with fewer than 10 minutes of data (after accounting for frames rejected due to head motion). ${ }^{45}$ This question was also addressed in terms of the amount of classifier error in the MLP method described above, where considerable error was found in data sets under 10 minutes of usable frames (Fig. 5). ${ }^{19}$ Therefore, we recommend obtaining at least 12 minutes of data, typically as two 6-minute runs, in the clinical setting, to allow an adequate margin of error for frames rejected due to scanner transients or head motion.

\section{Future Directions}

The clinical utilization of rs-fMRI and RSN mapping in neurosurgery has been limited to identifying regions to avoid during the resection of a lesion. However, emerging therapies in brain stimulation and therapeutic neuromodu-
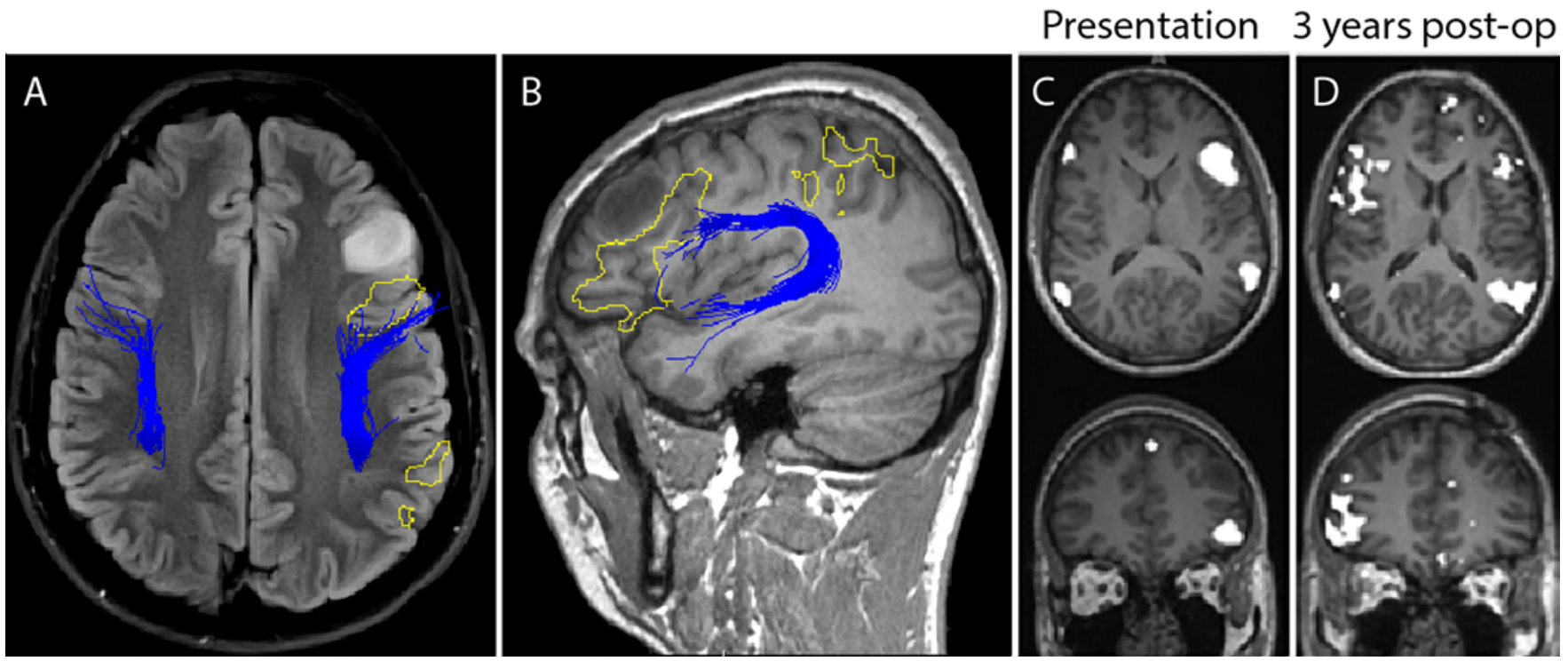

FIG. 4. Case 2. Left frontal glioma. FLAIR image (A) and T1-weighted MR image (B) demonstrating a left frontal lesion. Restingstate language network regions (yellow outlined regions) are seen in proximity to the $\mathrm{T} 1$ signal hypodense lesion. Tractography of the arcuate fasciculus (blue lines) colocalizes to language regions in the frontal lobe, posterior to the tumor. Language mapping (C) initially demonstrated a left-sided predominance. On repeat imaging at 3 years (D), the resting state demonstrates remapping of language network regions, contralateral to the tumor. 


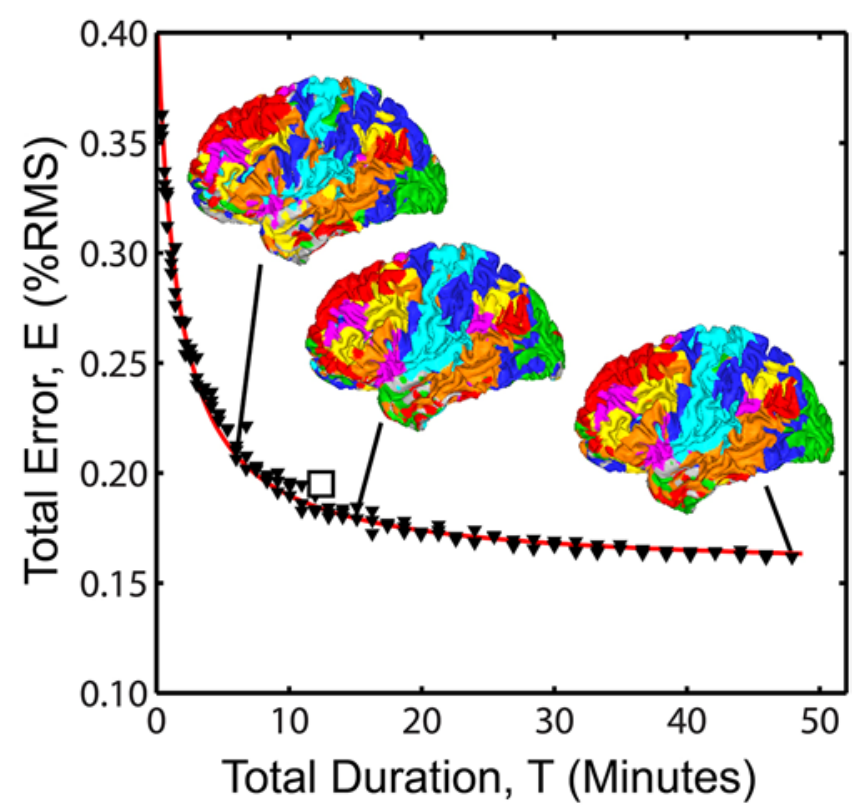

FIG. 5. Effect of total quantity of fMRI data on RSN classifier performance. The square marker indicates the recommended minimum clinical acquisition times (10-12 minutes). Adapted from Hacker CD, Laumann TO, Szrama NP, Baldassarre A, Snyder AZ, Leuthardt EC, et al: Resting state network estimation in individual subjects. Neuroimage 82:616-633, 2013; doi:10.1016/j.neuroimage.2013.05.108. Reproduced with permission of Elsevier.

lation create a new clinical need-specifically, preoperative guidance for explicit targeting of functional regions. Fox et al. systematically evaluated brain stimulation for multiple psychiatric and neurological diseases and found that sites for both invasive and noninvasive stimulation correspond to RSN topography..$^{11}$ For example, the dorsolateral prefrontal cortex (BA9/46) is a readily accessible target for both invasive and noninvasive therapies, given its location on the frontal convexity. Stimulation of BA46 for treatment-resistant depression was the first FDA-approved indication for transcranial magnetic stimulation (TMS), after multiple trials demonstrated significant efficacy. ${ }^{14,37} \mathrm{An}$ other major clinical target for the treatment of depression is the subgenual cingulate, which has been targeted with deep brain stimulation. ${ }^{29,30}$ BA46 is a region within the dorsal attention network, ${ }^{7}$ whereas the subgenual cingulate is a region within the default mode network. ${ }^{40}$ These regions are strongly and reliably anticorrelated; in fact, the efficacy of BA46 TMS for depression is related to the strength of fMRI anticorrelation with the subgenual cingulate. ${ }^{12}$

It is remarkable that rs-fMRI, a relatively new but fundamental observation of brain organization, recapitulates not only the topography of language and motor function as discussed previously, but also that of higher cognitive and emotional functions such as for mood and depression. ${ }^{16}$ There are two crucial implications for the design of neuromodulatory therapies. First, RSNs allow for an array of new potential targets. Examining regions anticorrelated with the subgenual cingulate (i.e., other regions in the dorsal attention network) provides a map of potential treatment targets throughout the brain. Second, RSNs are known to have significant interindividual variability, independent of anatomical variability. ${ }^{34}$ The current FDAapproved approach for targeting this region is to stimulate $5 \mathrm{~cm}$ anterior to the motor cortex (Fig. 6). The relationship between correlation strength and efficacy implies that individualized RSN mapping would be a better alternative to guide treatment planning. ${ }^{46}$
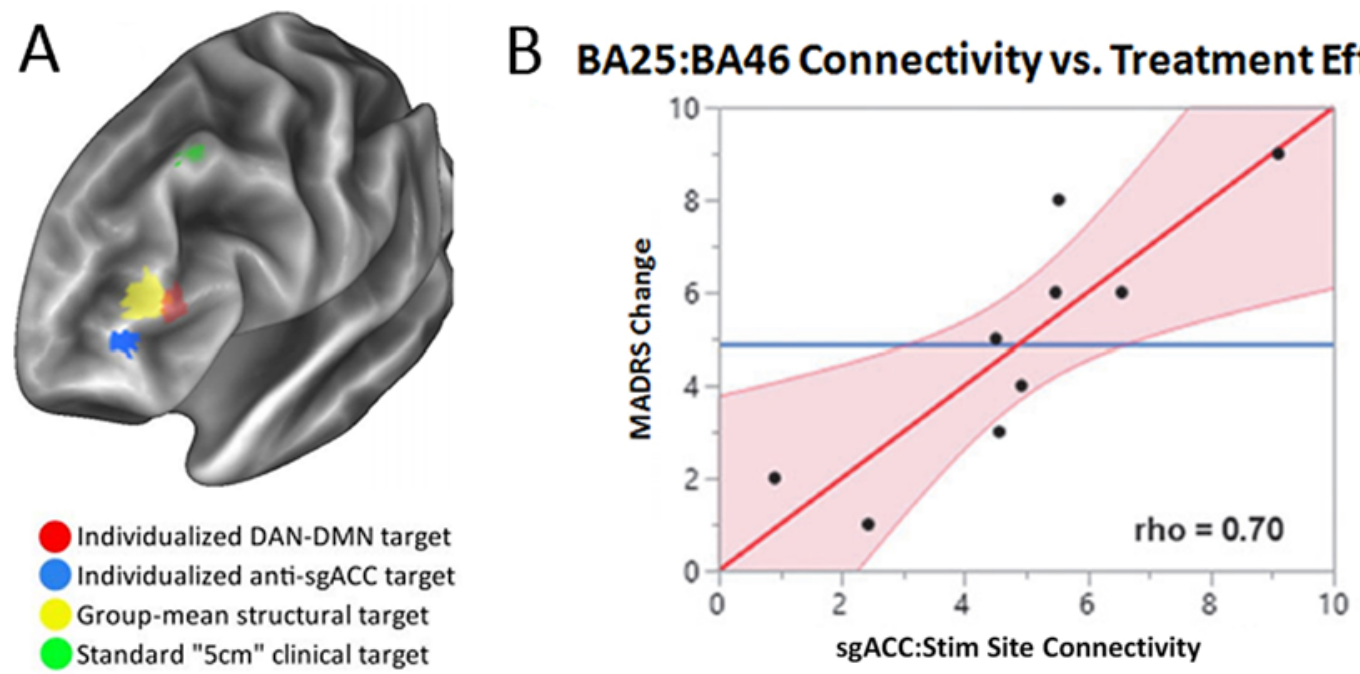

FIG. 6. RSN-guided TMS for the treatment of depression. A: Overlays indicate differences in therapeutic target. Note that a single patient's targeting using default mode network (DMN) and dorsal attention network (DAN) maps (red) may differ significantly from a group average target (yellow), and both of these differed substantially from the standard clinical targeting method (green). B: The correlation between the subgenual prefrontal cortex (BA25) and the dorsolateral prefrontal cortex (BA46) was significantly correlated to change in Montgomery-Asberg Depression Rating Scale (MADRS). Panel B from Siddiqi SH, Trapp NT, Hacker CD, Laumann TO, Kandala S, Hong X, et al: Repetitive transcranial magnetic stimulation with resting-state network targeting for treatment-resistant depression in traumatic brain injury: a randomized, controlled, double-blinded pilot study. J Neurotrauma 36:1361-1374, 2019. Reproduced with permission from Mary Ann Liebert, Inc. 


\section{Conclusions}

Functional mapping with rs-fMRI is a well-established practice that has only recently begun to enter the clinical realm. Initial experience in its neurosurgical application has shown great promise, but substantial work remains to establish its effect on clinical outcomes. Detailed neuropsychological assessments, combined with high-quality functional data, will lead to a more sophisticated understanding of critical functional regions and improved postoperative functional status.

\section{Acknowledgments}

The authors acknowledge the generous support from the National Institutes of Health (NIH R21 CA159470-01A1) and McDonnell Center Higher Brain Function, which made this research possible.

\section{References}

1. Beckmann CF, Smith SM: Probabilistic independent component analysis for functional magnetic resonance imaging. IEEE Trans Med Imaging 23:137-152, 2004

2. Bell AJ, Sejnowski TJ: An information-maximization approach to blind separation and blind deconvolution. Neural Comput 7:1129-1159, 1995

3. Binder JR, Gross WL, Allendorfer JB, Bonilha L, Chapin J, Edwards JC, et al: Mapping anterior temporal lobe language areas with fMRI: a multicenter normative study. Neuroimage 54:1465-1475, 2011

4. Biswal B, Yetkin FZ, Haughton VM, Hyde JS: Functional connectivity in the motor cortex of resting human brain using echo-planar MRI. Magn Reson Med 34:537-541, 1995

5. Biswal BB, Mennes M, Zuo XN, Gohel S, Kelly C, Smith SM, et al: Toward discovery science of human brain function. Proc Natl Acad Sci U S A 107:4734-4739, 2010

6. Briganti C, Sestieri C, Mattei PA, Esposito R, Galzio RJ, Tartaro A, et al: Reorganization of functional connectivity of the language network in patients with brain gliomas. AJNR Am J Neuroradiol 33:1983-1990, 2012

7. Corbetta M, Kincade JM, Ollinger JM, McAvoy MP, Shulman GL: Voluntary orienting is dissociated from target detection in human posterior parietal cortex. Nat Neurosci 3:292-297, 2000

8. Cordes D, Haughton VM, Arfanakis K, Wendt GJ, Turski PA, Moritz CH, et al: Mapping functionally related regions of brain with functional connectivity MR imaging. AJNR Am J Neuroradiol 21:1636-1644, 2000

9. Dierker D, Roland JL, Kamran M, Rutlin J, Hacker CD, Marcus DS, et al: Resting-state functional magnetic resonance imaging in presurgical functional mapping: sensorimotor localization. Neuroimaging Clin N Am 27:621-633, 2017

10. Duffau H, Capelle L, Sichez J, Faillot T, Abdennour L, Law Koune JD, et al: Intra-operative direct electrical stimulations of the central nervous system: the Salpêtrière experience with 60 patients. Acta Neurochir (Wien) 141:1157-1167, 1999

11. Fox MD, Buckner RL, Liu H, Chakravarty MM, Lozano AM, Pascual-Leone A: Resting-state networks link invasive and noninvasive brain stimulation across diverse psychiatric and neurological diseases. Proc Natl Acad Sci U S A 111:E4367-E4375, 2014

12. Fox MD, Buckner RL, White MP, Greicius MD, Pascual-Leone A: Efficacy of transcranial magnetic stimulation targets for depression is related to intrinsic functional connectivity with the subgenual cingulate. Biol Psychiatry 72:595-603, 2012

13. Fox MD, Snyder AZ, Vincent JL, Corbetta M, Van Essen
DC, Raichle ME: The human brain is intrinsically organized into dynamic, anticorrelated functional networks. Proc Natl Acad Sci U S A 102:9673-9678, 2005

14. George MS, Lisanby SH, Avery D, McDonald WM, Durkalski V, Pavlicova M, et al: Daily left prefrontal transcranial magnetic stimulation therapy for major depressive disorder: a sham-controlled randomized trial. Arch Gen Psychiatry 67:507-516, 2010

15. Gordon EM, Laumann TO, Gilmore AW, Newbold DJ, Greene DJ, Berg JJ, et al: Precision functional mapping of individual human brains. Neuron 95:791-807.e7, 2017

16. Greicius MD, Flores BH, Menon V, Glover GH, Solvason $\mathrm{HB}$, Kenna H, et al: Resting-state functional connectivity in major depression: abnormally increased contributions from subgenual cingulate cortex and thalamus. Biol Psychiatry 62:429-437, 2007

17. Gulati S, Jakola AS, Nerland US, Weber C, Solheim O: The risk of getting worse: surgically acquired deficits, perioperative complications, and functional outcomes after primary resection of glioblastoma. World Neurosurg 76:572-579, 2011

18. Håberg A, Kvistad KA, Unsgård G, Haraldseth O: Preoperative blood oxygen level-dependent functional magnetic resonance imaging in patients with primary brain tumors: clinical application and outcome. Neurosurgery 54:902-915, 2004

19. Hacker CD, Laumann TO, Szrama NP, Baldassarre A, Snyder AZ, Leuthardt EC, et al: Resting state network estimation in individual subjects. Neuroimage 82:616-633, 2013

20. Haglund MM, Berger MS, Shamseldin M, Lettich E, Ojemann GA: Cortical localization of temporal lobe language sites in patients with gliomas. Neurosurgery 34:567-576, 1994

21. Hornik K, Stinchcombe M, White H: Multilayer feedforward networks are universal approximators. Neural Netw 2:359366,1989

22. Jakola AS, Unsgård G, Solheim O: Quality of life in patients with intracranial gliomas: the impact of modern image-guided surgery. J Neurosurg 114:1622-1630, 2011

23. Keles GE, Chang EF, Lamborn KR, Tihan T, Chang CJ, Chang SM, et al: Volumetric extent of resection and residual contrast enhancement on initial surgery as predictors of outcome in adult patients with hemispheric anaplastic astrocytoma. J Neurosurg 105:34-40, 2006

24. Keles GE, Lamborn KR, Berger MS: Low-grade hemispheric gliomas in adults: a critical review of extent of resection as a factor influencing outcome. J Neurosurg 95:735-745, 2001

25. Khanna N, Altmeyer W, Zhuo J, Steven A: Functional neuroimaging: fundamental principles and clinical applications. Neuroradiol J 28:87-96, 2015

26. Lacroix M, Abi-Said D, Fourney DR, Gokaslan ZL, Shi W, DeMonte F, et al: A multivariate analysis of 416 patients with glioblastoma multiforme: prognosis, extent of resection, and survival. J Neurosurg 95:190-198, 2001

27. Laumann TO, Snyder AZ, Mitra A, Gordon EM, Gratton C, Adeyemo B, et al: On the stability of BOLD fMRI correlations. Cereb Cortex 27:4719-4732, 2017

28. Lee MH, Hacker CD, Snyder AZ, Corbetta M, Zhang D, Leuthardt EC, et al: Clustering of resting state networks. PLoS One 7:e40370, 2012

29. Lozano AM, Mayberg HS, Giacobbe P, Hamani C, Craddock RC, Kennedy SH: Subcallosal cingulate gyrus deep brain stimulation for treatment-resistant depression. Biol Psychiatry 64:461-467, 2008

30. Mayberg HS: Modulating dysfunctional limbic-cortical circuits in depression: towards development of brain-based algorithms for diagnosis and optimised treatment. Br Med Bull 65:193-207, 2003

31. McGirt MJ, Chaichana KL, Gathinji M, Attenello FJ, Than $\mathrm{K}$, Olivi A, et al: Independent association of extent of resec- 
tion with survival in patients with malignant brain astrocytoma. J Neurosurg 110:156-162, 2009

32. Meunier D, Lambiotte R, Bullmore ET: Modular and hierarchically modular organization of brain networks. Front Neurosci 4:200, 2010

33. Mitchell TJ, Hacker CD, Breshears JD, Szrama NP, Sharma M, Bundy DT, et al: A novel data-driven approach to preoperative mapping of functional cortex using resting-state functional magnetic resonance imaging. Neurosurgery 73:969-983, 2013

34. Mueller S, Wang D, Fox MD, Yeo BT, Sepulcre J, Sabuncu $\mathrm{MR}$, et al: Individual variability in functional connectivity architecture of the human brain. Neuron 77:586-595, 2013

35. Ojemann G, Ojemann J, Lettich E, Berger M: Cortical language localization in left, dominant hemisphere. An electrical stimulation mapping investigation in 117 patients. J Neurosurg 71:316-326, 1989

36. Ojemann GA: Functional mapping of cortical language areas in adults. Intraoperative approaches. Adv Neurol 63:155163,1993

37. O'Reardon JP, Solvason HB, Janicak PG, Sampson S, Isenberg KE, Nahas Z, et al: Efficacy and safety of transcranial magnetic stimulation in the acute treatment of major depression: a multisite randomized controlled trial. Biol Psychiatry 62:1208-1216, 2007

38. Poldrack RA, Laumann TO, Koyejo O, Gregory B, Hover A, Chen MY, et al: Long-term neural and physiological phenotyping of a single human. Nat Commun 6:8885, 2015

39. Power JD, Cohen AL, Nelson SM, Wig GS, Barnes KA, Church JA, et al: Functional network organization of the human brain. Neuron 72:665-678, 2011

40. Raichle ME, MacLeod AM, Snyder AZ, Powers WJ, Gusnard DA, Shulman GL: A default mode of brain function. Proc Natl Acad Sci U S A 98:676-682, 2001

41. Roland JL, Griffin N, Hacker CD, Vellimana AK, Akbari SH, Shimony JS, et al: Resting-state functional magnetic resonance imaging for surgical planning in pediatric patients: a preliminary experience. J Neurosurg Pediatr 20:583-590, 2017

42. Roland JL, Hacker CD, Snyder AZ, Shimony JS, Zempel JM, Limbrick DD, et al: A comparison of resting state functional magnetic resonance imaging to invasive electrocortical stimulation for sensorimotor mapping in pediatric patients. Neuroimage Clin 23:101850, 2019

43. Rosenblatt F: The perceptron: a probabilistic model for information storage and organization in the brain. Psychol Rev 65:386-408, 1958

44. Sanai N, Mirzadeh Z, Berger MS: Functional outcome after language mapping for glioma resection. $\mathbf{N}$ Engl J Med 358:18-27, 2008

45. Seitzman BA, Snyder AZ, Leuthardt EC, Shimony JS: The state of resting state networks. Top Magn Reson Imaging 28:189-196, 2019

46. Siddiqi SH, Trapp NT, Hacker CD, Laumann TO, Kandala S, Hong X, et al: Repetitive transcranial magnetic stimulation with resting-state network targeting for treatment-resistant depression in traumatic brain injury: a randomized, con- trolled, double-blinded pilot study. J Neurotrauma 36:13611374, 2019

47. Smith SM, Fox PT, Miller KL, Glahn DC, Fox PM, Mackay $\mathrm{CE}$, et al: Correspondence of the brain's functional architecture during activation and rest. Proc Natl Acad Sci U S A 106:13040-13045, 2009

48. Stummer W, Pichlmeier U, Meinel T, Wiestler OD, Zanella F, Reulen HJ: Fluorescence-guided surgery with 5-aminolevulinic acid for resection of malignant glioma: a randomised controlled multicentre phase III trial. Lancet Oncol 7:392401, 2006

49. Traut T, Sardesh N, Bulubas L, Findlay A, Honma SM, Mizuiri D, et al: MEG imaging of recurrent gliomas reveals functional plasticity of hemispheric language specialization. Hum Brain Mapp 40:1082-1092, 2019

50. van den Heuvel MP, Stam CJ, Boersma M, Hulshoff Pol HE: Small-world and scale-free organization of voxel-based resting-state functional connectivity in the human brain. Neuroimage 43:528-539, 2008

51. Wagner K, Hader C, Metternich B, Buschmann F, Schwarzwald R, Schulze-Bonhage A: Who needs a Wada test? Present clinical indications for amobarbital procedures. J Neurol Neurosurg Psychiatry 83:503-509, 2012

52. Wig GS, Laumann TO, Cohen AL, Power JD, Nelson SM, Glasser MF, et al: Parcellating an individual subject's cortical and subcortical brain structures using snowball sampling of resting-state correlations. Cereb Cortex 24:2036-2054, 2014

53. Yeo BT, Krienen FM, Eickhoff SB, Yaakub SN, Fox PT, Buckner RL, et al: Functional specialization and flexibility in human association cortex. Cereb Cortex 25:3654-3672, 2015

54. Yeo BT, Krienen FM, Sepulcre J, Sabuncu MR, Lashkari D, Hollinshead M, et al: The organization of the human cerebral cortex estimated by intrinsic functional connectivity. J Neurophysiol 106:1125-1165, 2011

\section{Disclosures}

Dr. Kim reports receiving research support for the present study from Monteris Medical and from Stryker. Dr. Leuthardt reports holding stock in NeuroLutions, General Sensing, and OsteoVantage.

\section{Author Contributions}

Conception and design: Hacker, Roland, Shimony, Leuthardt. Acquisition of data: all authors. Analysis and interpretation of data: all authors. Drafting the article: Hacker, Leuthardt. Critically revising the article: all authors. Reviewed submitted version of manuscript: all authors. Approved the final version of the manuscript on behalf of all authors: Hacker. Statistical analysis: Hacker. Administrative/technical/material support: Hacker.

\section{Correspondence}

Carl D. Hacker: Washington University School of Medicine, St. Louis,MO.hackerc@wustl.edu. 